\title{
CONVERGENCE THEOREMS FOR BANACH SPACE VALUED INTEGRABLE MULTIFUNCTIONS
}

\author{
NIKOLAOS S. PAPAGEORGIOU \\ Department of Mathematics \\ University of California \\ Davis, California 95616 \\ (Received May 21, 1986)
}

\begin{abstract}
In this work we generalize a result of Kato on the pointwise behavior of a weakly convergent sequence in the Lebesgue-Bochner spaces $L_{X}^{P}(\Omega)(1 \leq p \leq \infty)$. Then we use that result to prove Fatou's type lemmata and dominated convergence theorems for the Aumann integral of Banach space valued measurable multifunctions. Analogous convergence results are also proved for the sets of integrable selectors of those multifunctions. In the process of proving those convergence theorems we make some useful observations concerning the Kuratowski-Mosco convergence of sets.
\end{abstract}

KEY WORDS AND PHRASES. Convergence, measurable multifunctions, nonatomic. 1980 AMS SUBJECT CLASSIFICATION CODE. 28A45, 46G10.

\section{INTRODUCTION.}

In [1] Schmeidler motivated from problems in mathematical economics, proved a set valued version of Fatou's lemma, for multifunctions taking values in $\mathbb{R}^{\text {n }}$ A different proof and some additional results in this direction were obtained later by Hildenbrand and Mertens [2].

Finally Artstein in [3] provided the sharpest version of that result. However a11 the above authors apparently were unaware of an earlier analogous result of Kato [4], for Banach space valued functions. The purpose of this note is to significantly extend the result of Kato [4], use that extension to prove a Fatou's lemma for Banach space valued multifunctions, extending this way the works of Schmeidler [1], Hildenbrand-Mertens [2] and Artstein [3] and finally prove a dominated convergence theorem for Banach space valued multifunctions. Then we obtain analogous convergence results for the sets of Bochner integrable selectors of the multifunctions. Our results can have important applications in optimization, optimal control, differential inclusions, abstract evolution equations and mathematical economics.

2. PRELIMINARIES.

Let $(\Omega, \Sigma, \mu)$ be a complete, $\sigma$-finite measure space and $X$ a separable Banach space, with $\mathrm{X}^{*}$ being its topological dual. We will use the following notations:

$$
\underset{f(C)}{P(X)}=\{A \subseteq X: \text { nonempty, closed, (convex) }\}
$$


$\underset{w k(c)}{P(x)}=\{A \leq X:$ nonempty, w-compact, (convex) $\}$

For $A \in 2^{X} \backslash\{\phi\}$, we set $|A|=\sup _{a \in A}|| a||$, by $d_{A}(\cdot)$ we denote the distance function from $A$ i.e. for all $x \in x, d_{A}(x)=\underset{a \varepsilon A}{\inf }\|x-a\|$ and by $\sigma_{A}(\cdot)$ the support function of A i.e. for all $x^{*} \varepsilon x^{*}, \sigma_{A}\left(x^{*}\right)=\sup _{a \in A}\left(x^{*}, a\right)$.

A multifunction $F: \Omega+P_{f}(X)$ is said to be measurable if it satisfies any of the following equivalent conditions:

i) for all $x \in X, \omega \rightarrow \underset{F(\omega)}{(x)}$ is measurable

ii) there exists a sequence $\left\{f_{n}(\cdot)\right\}_{n>1}$ of measurable functions s.t. $F(\omega)=c 1\left\{f_{n}(\omega)\right\}_{n \geq 1}$ for all $\omega \in \Omega \quad$ (Castaing's representation).

iii) $\mathrm{GrF}=\{(\omega, \mathrm{x}) \in \Omega \times \mathrm{XX}: \mathrm{x} \in \mathrm{F}(\omega)\} \in \sum \mathrm{XB}(\mathrm{X})$, where $\mathrm{B}(\mathrm{X})$ is the Borel o-field of $X$ (graph measurability).

We denote by $S_{F}^{1}$ the set of all selectors of $F(\cdot)$ that belong to the LebesgueBochner space $L_{X}^{1}(\Omega)$ 1.e. $S_{F}^{1}=\left\{f(\cdot) \varepsilon L_{X}^{1}(\Omega): f(\omega) \varepsilon F(\omega) \mu-a . e.\right\}$. It is easy to see

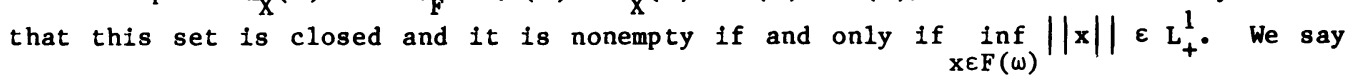
that $F: X \rightarrow P_{f}(X)$ is integrably bounded if it is measurable and $|F(\cdot)| \varepsilon L_{+}^{1}$. Using the set $S_{F}^{1}$, we can define a set valued integral for $F(\cdot)$ as follows: $\int_{\Omega} F(\omega) \mathrm{d} \mu(\omega)=\left\{\int_{\Omega} f(\omega) \mathrm{d} \mu(\omega): f(\cdot) \varepsilon s_{F}^{1}\right\}$. This integral is known as Aumann's integral. If $\left\{A_{n}\right\}_{n \geq 1}$ are nonempty subsets of $x$, we define

$$
s-\frac{\lim }{n \rightarrow \infty} A_{n}=\left\{x \in x: x=s-\lim x_{n}, x_{n} \varepsilon A_{n}, n \geq 1\right\}
$$

and $w-\overline{\lim }_{n \rightarrow \infty} A_{n}=\left\{x \in x: x-1 \lim x_{k}, x_{k} \varepsilon A_{n_{k}}, k \geq 1\right\}$.

We say that the $A_{n}^{\prime} s$ converge to $A$ in the Kuratowski-Mosco sense (denoted by $\left.A_{n} \stackrel{K-M}{\rightarrow} A\right)$ if and only if $w-\overline{1 I m} A_{n}=A=s-\underline{\lim } A_{n}$. For more details we refer to the nice works of Mosco [5], [6] and of Salinetti and Wets [7], [8] and [9].

3. CONVERGENCE RESULTS FOR THE AUMANN INTEGRAL.

In this section our goal is to prove a Fatou's lemma and a dominated convergence theorem for the Aumann integral. We start with an interesting observation concerning the $w-\overline{1 m}$ of a sequence of nonempty sets. Assume that $X$ is a Banach space.

PROPOSITION 3.1. If for all $n \geq 1 \quad A_{n} \neq \phi$ and $A_{n} \subseteq G$ where $G \varepsilon P_{w k}(x)$

then for all $x^{*} \varepsilon x^{*}, \overline{1 i m} \sigma_{A_{n}}\left(x^{*}\right) \leq \sigma \frac{\left(x^{*}\right)}{w-1 i m} A_{n}$.

PROOF. FIx $x^{*} \varepsilon x^{*}$ and let $x_{n} \varepsilon A_{n}$ s.t. $\left(x^{*}, x_{n}\right)=\sigma_{A_{n}}\left(x^{*}\right)$. Let $\left\{x_{k}\right\}{ }_{k}$ be a subsequence of $\left\{x_{n}\right\}_{n \geq 1}$ s.t. $\left(x^{*}, x_{k}\right) \rightarrow \overline{\operatorname{Iim}}_{A_{n}}\left(x^{*}\right)$ as $k+\infty_{n}$. Since $\left\{x_{n}\right\}_{n \geq 1} \subseteq G$, 
invoking the Eberlein-Smulian theorem and by passing to a subsequence if necessary, we may assume that $x_{k} \stackrel{-w}{\rightarrow} x$.

Then $x \in w-\overline{1 i m} A_{n} \Rightarrow\left(x^{*}, x\right) \leq \sigma \frac{\left(x^{*}\right)}{w-\overline{\lim } A_{n}} \Rightarrow \overline{\lim \sigma_{A_{n}}\left(x^{*}\right) \leq \sigma} \frac{\left(x^{*}\right)}{w-\overline{1 i m} A_{n}}$.

Q.E.D.

This leads us to the following interesting theorem that generalizes significantly an earlier result of Kato [4], who had $X$ to be reflexive with a uniformly convex dual, $1<\mathrm{p}<\infty$ and the sequence of vector valued functions was uniformly bounded.

Here $(\$ 2, \Sigma, \mu)$ is a measure space, $X$ a Banach space and $1 \leq p<\infty$.

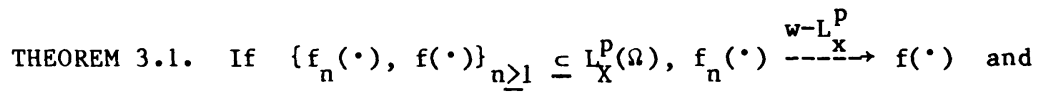
$\mathrm{f}_{\mathrm{n}}(\omega) \varepsilon G(\omega) \mu-\mathrm{a}$. e. where $G(\omega) \varepsilon \mathrm{P}_{\mathrm{wk}}(\mathrm{X}) \mu-$-a.e.

then $f(\omega) \varepsilon \overline{\operatorname{conv}} w-\overline{\lim }\left\{f_{n}(\omega)\right\}_{n \geq 1}^{\mu-a . e .}$

PROOF. From Mazur's lemma we know that for all $k \geq 1$

$$
f(w) \varepsilon \overline{\operatorname{conv}} \underset{n \geq k}{\bigcup} f_{n}(\omega) \mu-a . e .
$$

Let $x^{*} \varepsilon x^{*}$. Then for all $k \geq 1$ we have:

$$
\begin{aligned}
& \left(x^{*}, f(\omega)\right) \leq \sigma \frac{\left(x^{*}\right)}{\operatorname{conv}} \underset{n \geq k}{\bigcup} f_{n}(\omega)=\sigma \underset{n \geq k}{U\left(x^{*}\right)} f_{n}(\omega)=\sup _{n \geq k}\left(x^{*}, f_{n}(\omega)\right) \mu-a \cdot e . \\
& \Rightarrow\left(x^{*}, f(\omega)\right) \leq \overline{1 i m}\left(x^{*}, f_{n}(\omega)\right)=\overline{11 m} \sigma \underset{\left\{f_{n}(\omega)\right\}}{\left(x^{*}\right)} \mu-a \cdot e .
\end{aligned}
$$

Using proposition 3.1 we get that

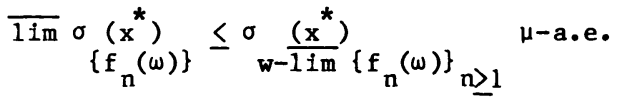

$$
\begin{aligned}
& \Rightarrow\left(x^{*}, f(\omega)\right) \leq \sigma \frac{\left(x^{*}\right)}{w-1 i m}\left\{f_{n}(\omega)\right\}{ }_{n \geq 1} \mu-a_{\cdot} e . \\
& \Rightarrow f(w) \varepsilon \overline{\operatorname{conv}} w-\overline{\lim }\left\{f_{n}(\omega)\right\}_{n \geq 1}^{\mu-a . e .}
\end{aligned}
$$

Q.E.D.

Having this theorem we can have the w- $\overline{1 \mathrm{im}}$ version of Fatou's lemma for the Aumann integral.

Now $(\Omega, \Sigma, \mu)$ is a nonatomic, $\sigma$-finite, complete measure space and $X$ a separable Banach space. 
THEOREM 3.2. If $F_{n}: s i P_{f}(X)$ are measurable multifunctions s.t. for all $\mathrm{n} \geq 1, \mathrm{~F}_{\mathrm{n}}(\omega) \leq \mathrm{G}(\omega) \mu-\mathrm{a} \cdot \mathrm{e}$. where $\mathrm{G}: \Omega \rightarrow \mathrm{P}_{\mathrm{wkc}}(\mathrm{X})$ is Integrably bounded and $\omega \rightarrow \omega-\overline{1 i m} F_{n}(\omega)$ is measurable

$$
\text { then } w-\overline{1 i m} \int_{\Omega} F_{n}(\omega) d \mu(\omega) \leq c 1 \int_{\Omega} w-\overline{1 i m} F_{n}(\omega) d \mu(\omega)
$$

PROOF. Let $x \in w-\overline{1 i m} \int_{\Omega} F_{n}(\omega) \mathrm{d} \mu(\omega)$. Then there exist $x_{k} \varepsilon \int_{\Omega} F_{n_{k}}(\omega) d \mu(\omega)$ s.t. $x_{k} \stackrel{\Omega_{w}}{\rightarrow} x$. From the definition of the Aumann integral, we know that there exist $f_{k}(\cdot) \varepsilon s_{F_{n_{k}}}^{1}$ s.t. $x_{k}=\int_{\Omega} f_{k}(\omega) d \mu(\omega)$. But $s_{F_{n_{k}}}^{1} s_{G}^{1}$ and the latter is $w$-compact in $\mathrm{L}_{\mathrm{X}}^{1}(\Omega) \quad[10]$. So by passing if necessary to a further subsequence, we may assume that $f_{k}(\cdot) \stackrel{w-L^{l} X}{--} f(\cdot) \varepsilon s_{G}^{l}$. Hence $x=\int_{\Omega} f(\omega) \operatorname{d\mu }(\omega)$. But from theorem 3.1 we know that $f(\omega) \varepsilon \overline{\operatorname{conv}} w-\overline{1 i m}\left\{f_{n}(\omega)\right\}{ }_{n \geq 1} \mu-a_{. e} \Rightarrow f(\omega) \varepsilon \overline{\operatorname{conv}} w-\overline{11 m}$ $F_{n}(\omega) \mu-a . e .=>x \in \int_{\Omega} \overline{\operatorname{conv}} w-\overline{1 i m} F_{n}(\omega) d \mu(\omega)$. Since by hypothesis $\omega+\omega-\overline{1 i m} F_{n}(\omega)$ is graph measurable and $\mu(\cdot)$ is nonatomic, we have that $c 1 \int_{\Omega} \omega-\overline{\lim } \mathrm{F}_{\mathrm{n}}(\omega) \mathrm{d} \mu(\omega)=\int_{\Omega} \overline{\operatorname{conv}} \omega-\overline{\lim } \mathrm{F}_{\mathrm{n}}(\omega) \mathrm{d} \mu(\omega)$. Thus finally we have that $x \in \operatorname{cl} \int_{\Omega} w-\overline{\lim } F_{n}(\omega) \mathrm{d} \mu(\omega)$, which proves Fatou's lemma for the weak limit superior.

Q.E.D.

Next we will prove the s-11m version of Fatou's lemma. This can be achieved under less restrictive hypotheses on the sequence $\left\{F_{n}\left({ }^{\bullet}\right)\right\}{ }_{n \geq 1}{ }^{\bullet}$

Here $(\Omega, \Sigma, \mu)$ is a complete, $\sigma$-finite measure space and $X$ a separable Banach space.

THEOREM 3.3. If $F_{n}: \delta \rightarrow 2^{X} \backslash\{\phi\}$ are integrab1y bounded and $\left.\left\{\left|F_{n}(\cdot)\right|\right\}\right\}_{n \geq 1}$ is uniformly Integrable

$$
\text { then } \int_{\Omega} s-\underline{1 i m} F_{n} f(\omega) \mathrm{d} \mu(\omega) \leq s-1 \operatorname{im} \int_{\Omega} F_{n}(\omega) \mathrm{d} \mu(\omega) .
$$

PROOF. Let $\quad x \in \int_{\Omega}$ s- $\lim F_{n} f(\omega) \mathrm{d} \mu(\omega)$. Then $x=\int_{\Omega} f(\omega) \mathrm{d} \mu(\omega)$ with $f(\cdot) \varepsilon$ $\mathrm{S}_{\mathrm{s}-1 \text { im }}^{1} \mathrm{~F}_{\mathrm{n}}$. Now consider the multifunctions $\mathrm{L}_{\mathrm{n}}(\omega)=$ $\left.\left\{x \in F_{n}(\omega): d_{n}(\omega)(\omega)\right) \leq\|x-f(\omega)\|+\frac{1}{n}\right\}$. Because the function $(\omega, x) \rightarrow d(x)$ is Caratheodory, it is superpositionally measurable and so $F_{n}(\omega)$

$\omega \rightarrow \underset{F_{n}}{(\omega)}\left(\underset{f(\omega))}{n}\right.$ is measurable. Then $(\omega, x)+\underset{F_{n}(\omega)}{(f(\omega))}-\|x-f(\omega)\|$ is a Caratheodory 


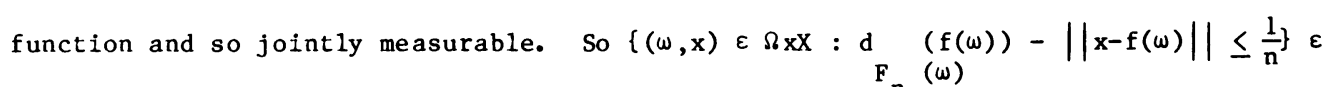

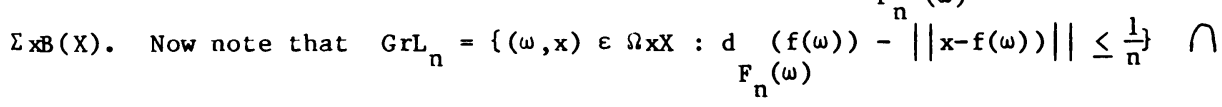
$\mathrm{GrF}_{\mathrm{n}} \varepsilon \sum \mathrm{XB}(\mathrm{X})$. Apply Aumann's selection theorem to find $\mathrm{f}_{\mathrm{n}}: \Omega+\mathrm{X}$ measurable s.t. $f_{n}(\omega) \varepsilon L_{n}(\omega)$ for all $\omega \varepsilon \Omega$. From the definition of $s-1$ im $F_{n}(\omega)$ [11] we know that $\mathrm{d}_{\mathrm{F}_{\mathrm{n}}(\omega)}(\mathrm{f}(\omega)) \rightarrow 0 \mu-\mathrm{a}$. e. So || $\mathrm{f}_{\mathrm{n}}(\omega)-\mathrm{f}(\omega)||+0 \mu-\mathrm{a} \cdot$ e. $\Rightarrow \int_{\Omega} \mathrm{f}_{\mathrm{n}}(\omega) \mathrm{d} \mu(\omega)=\mathrm{x}_{\mathrm{n}}-\underline{s}_{\rightarrow} \rightarrow$ $\int_{\delta \Omega} f(\omega) \mathrm{d} \mu(\omega)=x$ and $x_{n} \varepsilon \int_{\Omega \Omega} F_{n}(\omega) d \mu(\omega) \Rightarrow x \varepsilon s-\underline{1 i m} \int_{\Omega} F_{n}(\omega) d \mu(\omega)$. Hence Fatou's lemma follows.

Q.E.D.

REMARK. From Kuratowski [11], we know that an equivalent definition of s-1im $F_{n}(\omega)$ is: $s-\underline{1 i m} F_{n}(\omega)=\left\{x \in X: \underset{n \rightarrow \infty}{\lim } \underset{F_{n}}{(x)} \underset{(\omega)}{(x)}=0\right\}$ and that $s-\underline{1 i m} F_{n}(\omega)$ is a closed set. Note that $(\omega, x)+d(x)$ being Caratheodory it is jointly measurable $F_{n}(\omega)$

and then so is 1 im $d \quad(x)$. Hence $\{(\omega, x) \varepsilon \Omega x X: 1 i m d \quad(x)=0\} \varepsilon \Sigma \times B(X) \Rightarrow$ $\mathrm{n}_{n \rightarrow \infty} \mathrm{F}_{\mathrm{n}}(\omega) \quad \mathrm{n}^{+\infty} \mathrm{F}_{\mathrm{n}}(\omega)$

$\operatorname{Gr}\left(s-\underline{1 \text { im }} F_{n}(\cdot)\right) \varepsilon \Sigma \times B(X) \Rightarrow \omega \rightarrow s-1$ im $F_{n}(\omega)$ is measurable.

Combining the two Fatou's lemmata we can have a dominated convergence theorem for the Aumann integral.

So assume that $(\Omega, \Sigma, \mu)$ is nonatomic, complete, $\sigma-f i n i t e$ mesure space and $X$ a separable Banach space.

THEOREM 3.4. If $F_{n}: \Omega+P_{f}(X)$ are measurable multifunctions s.t. $F_{n}(\omega) \subseteq$ $G(\omega) \mu-a . e$. with $G: \Omega \rightarrow P_{w k c}(X)$ integrably bounded and $F_{n}(\omega) \stackrel{K}{-}-\underline{M} \rightarrow F(\omega) \mu-a . e$.

$$
\text { then } \int_{\Omega} F_{n}(\omega) \mathrm{d} \mu(\omega) \stackrel{\text { K-M }}{\rightarrow} \rightarrow \mathrm{cl} \int_{\Omega} F(\omega) \mathrm{d} \mu(\omega)
$$

PROOF. This follows from theorem 3.2 and 3.3 if we recall that $F_{n}(\omega) \rightarrow$ $F(\omega) \mu-a . e . \Leftrightarrow w-\overline{1 i m} F_{n}(\omega)=F(\omega)=\sin F_{n}(\omega)$ and $F(\cdot)$ is closed valued and measurable.

Q.E.D.

REMARK. If we assume that $F\left(^{\cdot}\right)$ is convex valued (which is the case if the $F_{n}^{\prime} s$ are) then we have that $\int_{\Omega} F_{n}(\omega) \mathrm{d} \mu(\omega) \stackrel{\mathrm{K}-M}{\rightarrow} \int_{\Omega} F(\omega) \mathrm{d} \mu(\omega)[10]$. Furthermore in this case we can relax the nonatomicity hypothesis on $\mu(\cdot)$.

We will close this section with a dominated convergence theorem for the Hausdorf $f$ metric $h(\cdot, \cdot)$ on $\mathrm{P}_{\mathrm{f}}(\mathrm{X})$.

Let $(\Omega, \Sigma, \mu)$ be a complete, $\sigma$-finite measure space and $X$ a separable Banach space.

THEOREM 3.5. If $F_{n}: \Omega \rightarrow P_{f}(X)$ are measurable multifunctions, $\left\{\left|F_{n}(\cdot)\right|\right\}_{n} \geq 1$ is uniformly integrable and $F_{n}(\omega)-\underline{h} \rightarrow F(\omega)$ in measure 
then $c 1 \int_{\Omega \Omega} F_{n}(\omega) \mathrm{d} \mu(\omega) \stackrel{h}{\rightarrow} c 1 \int_{\Omega \Omega} F(\omega) \mathrm{d} \mu(\omega)$.

PROOF. Recall that $h\left(c 1 \int_{\Omega} F_{n}(\omega) \mathrm{d} \mu(\omega), c 1 \int_{\Omega} F(\omega) \mathrm{d} \mu(\omega)\right) \leq \int_{\Omega} h\left(F_{n}(\omega), F(\omega)\right) \mathrm{d} \mu(\omega)$. Also $h\left(F_{n}(\omega), F(\omega)\right) \leq\left|F_{n}(\omega)\right|+|F(\omega)|$. Then using the extended dominated convergence theorem [12], we get $\int_{\Omega} h\left(F_{n}(\omega), F(\omega)\right) d \mu(\omega)+0 \Rightarrow h\left(c 1 \int_{\Omega} F_{n}(\omega) d \mu(\omega)\right.$, cl $\left.\int F(\omega) \mathrm{d} \mu(\omega)\right) \rightarrow 0$ as $n \rightarrow \infty$.

$$
\text { Q..E.D. }
$$

4. CONVERGENCE RESULTS FOR THE SETS OF INTEGRABLE SELECTORS.

In this section we prove analogous convergence theorems for the sets $s_{F_{n}}^{1}$.

As before we will start with two Fatou's type theorems. But first we need the following auxillary result about the Kuratowski-Mosco convergence of sets.

Here $X$ is any Banach space.

PROPOSITION 4.1. If for all $x^{*} \varepsilon x^{*}, \overline{11 m} \sigma_{A_{n}}\left(x^{*}\right) \leq \sigma_{A}\left(x^{*}\right)$

then $w-\overline{1 i m} A_{n} \subseteq \overline{\operatorname{conv}} A$

PROOF. Let $x \in w-\overline{\lim } A_{n}$. Then there exist $x_{k} \varepsilon A_{n_{k}}$ s.t. $x_{k}-\underline{w} \rightarrow x_{*}$ So for

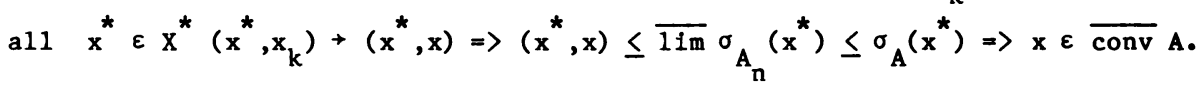

\section{Q.E.D.}

Now we are ready for the first Fatou's type convergence result. So let $(\Omega, \Sigma, \mu)$ be a complete, $\sigma$-finite measure space and $X$ a separable Banach space. THEOREM 4.1. If $F_{n}: \Omega \rightarrow P_{f}(X)$ are measurable multifunctions s.t. $\left\{\left|F_{n}(\cdot)\right|\right\}_{n \geq 1}$ is uniformly integrable and s-1im $F_{n}(\omega) \neq \phi \mu-a . e$.

$$
\text { then } S_{s-1 \text { im }}^{1} F_{n} \subseteq \underline{s-1 \text { im }} S_{F_{n}}^{1}
$$

PROOF. Let $u(\cdot) \in \mathrm{L}_{\mathrm{X}}^{1}(\Omega)$. Then we have:

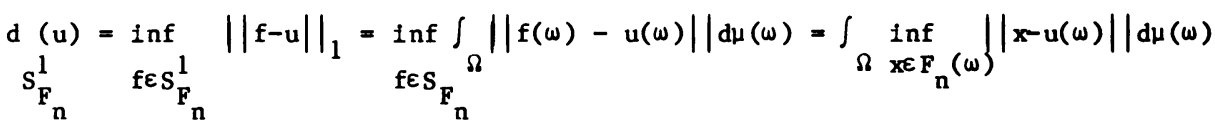

$$
\begin{aligned}
& \left.=\int_{\Omega} \underset{F_{n}}{d}(w)(w)\right) d \mu(w)
\end{aligned}
$$

So using Fatou's lemma [12] we get that: 


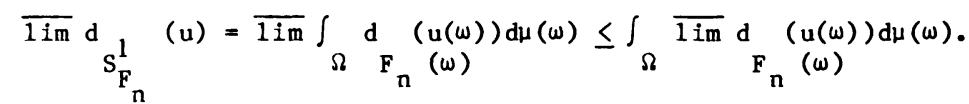

But from theorem 2.2 (i) of Tsukada [13] we have that for all $\omega \varepsilon \Omega$

$$
\begin{aligned}
& \overline{\lim }_{\mathrm{F}_{\mathbf{n}}(\omega)} \underset{(u(\omega))}{(\omega)} \leq \underset{\mathrm{s}-\underline{\lim } \mathrm{F}_{\mathbf{n}}(\omega)}{(u(\omega))}
\end{aligned}
$$

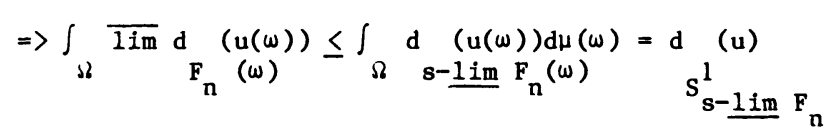

$$
\begin{aligned}
& \Rightarrow \overline{1 i m} \mathrm{~d} \quad(\mathrm{u}) \leq \mathrm{d} \quad(\mathrm{u}) \quad \text {. } \\
& s_{F_{n}}^{1} \quad s_{s-1 \text { im }}^{1} F_{n}
\end{aligned}
$$

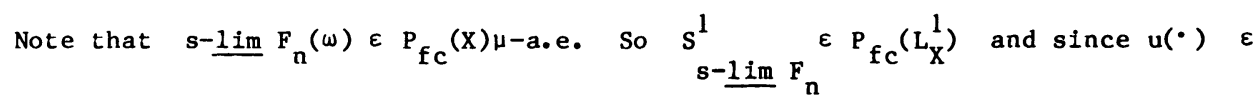
$L_{X}^{1}(\Omega)$ was arbitrary we can apply theorem 2.2 (ii) of Tsukada [13] and conclude that

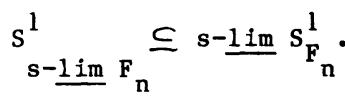

Q.E.D.

We have the analogous result for $w-\overline{1 i m}$. The assumptions on the spaces $(\Omega, \Sigma, \mu)$ and $X$ remain the same.

THEOREM 4.2. If $F_{\mathrm{n}}: \Omega \rightarrow \mathrm{P}_{\mathrm{fc}}(\mathrm{X})$ are measurable multifunctions s.t. for all $\mathrm{n} \geq 1 \mathrm{~F}_{\mathrm{n}}(\omega) \subseteq \mathrm{G}(\omega) \phi-$ a.e. where $\mathrm{G}: \Omega \rightarrow \mathrm{P}_{\text {wkc }}(\mathrm{X})$ is integrably bounded and $\omega+\omega-\overline{1 i m} F_{n}(\omega)$ is graph measurable

$$
\begin{gathered}
\text { then } w-\overline{1 i m} S_{F_{n}}^{1} \subseteq \overline{\operatorname{conv}} s_{w-\overline{1 i m} F_{n}}^{1} \\
\text { If in addition } w-\overline{1 i m} F_{n}(\omega) \varepsilon P_{f c}(X) \text { for all } \omega \varepsilon \Omega \\
\text { then } w-\overline{1 i m} s_{F_{n}}^{1} \subseteq S_{w-1 i m}^{1} F_{n} .
\end{gathered}
$$

PROOF. From the Dinculeanu-Foias theorem [14], we know that $\left(L_{X}^{1}\right)^{*}=L_{X^{*}}^{\infty}$. Let $\mathrm{u}\left({ }^{\cdot}\right) \in \mathrm{L}_{\mathrm{X}_{*}^{*}}^{\infty}{ }_{\mathrm{w}}$. Then we have:

$$
\begin{aligned}
& \sigma_{F_{n}^{1}}(u)=\sup _{f(\cdot) \varepsilon S_{F_{n}}^{1}} \int_{\Omega}(u(\omega), f(\omega)) d \mu(\omega)= \\
& \int_{\delta \Omega} \sup _{x \in F_{n}(\omega)}(u(\omega), x) d \mu(\omega)=\int_{\Omega} \sigma F_{n}(u(\omega)) d \mu(\omega) .
\end{aligned}
$$


Then using Fatou's lemma we get that

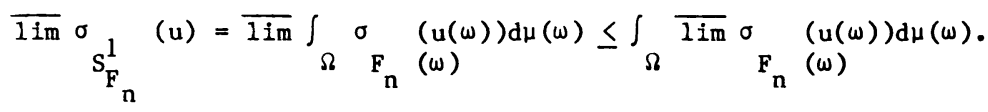

But from proposition 3.1 we know that for all $\omega \varepsilon \Omega$

$$
\overline{\operatorname{Iim} \sigma} \sigma_{n}(\omega(\omega)) \leq \sigma \frac{(u(\omega))}{w-1 i m} F_{n}(\omega)
$$

and since $w-\overline{\lim } \mathrm{F}_{\mathrm{n}}(\omega)$ is by hypothesis graph measurable we have that:

$$
\int_{\Omega \Omega} \sigma \frac{(u(\omega)) d \mu(\omega)}{w-1 i m} F_{n}(\omega)=\sigma \frac{(u)}{s_{w-1 i m}^{1}}
$$

So finally we have that:

$$
\overline{\lim } \sigma_{\mathrm{F}_{\mathrm{n}}^{1}}(\mathrm{u}) \leq \sigma \mathrm{s}_{\mathrm{w}-\overline{\lim } \mathrm{F}_{\mathrm{n}}}
$$
Since this is true for every $u(\cdot) \varepsilon \mathrm{L}_{\mathrm{X}_{*}^{*}}^{\infty}=\left(\mathrm{L}_{\mathrm{X}}^{1}\right)^{*}$, from proposition 4.1 we
conclude that

$$
\mathrm{w}-\overline{\lim } \mathrm{S}_{\mathrm{F}_{\mathrm{n}}}^{1} \subseteq \overline{\operatorname{conv}} \mathrm{s}_{\mathrm{w}}^{1} \overline{\text { Iim } \mathrm{F}_{\mathrm{n}}}
$$

If in addition, $w-\overline{1 \mathrm{Im}} F_{n}(\cdot)$ is $P_{f c}(X)$-valued, then $s^{1}{ }_{w}-\overline{1 I m} F_{n}$ Is convex and of course closed and so $w-\overline{1 i m} s_{F_{n}}^{1} \subseteq s_{w-1 i m}^{1} F_{n}$.

$$
\text { Q.E.D. }
$$

Combining theorems 4.1 and 4.2 we can have a dominated convergence theorem for the sequence $\left\{S_{F_{n}}^{1}\right\}_{n \geq 1}$. Our assumptions on $(\Omega, \Sigma, \mu)$ and $X$ remain as before.

THEOREM 4.4. If $F_{n}: \Omega+P_{f c}(X)$ are measurable multifunctions s.t. for all $\mathrm{n} \geq 1 \mathrm{~F}_{\mathrm{n}}(\omega) \subseteq \mathrm{G}(\omega) \mu-\mathrm{a}$. e. where $\mathrm{G}: \Omega \rightarrow \mathrm{P}_{\text {wkc }}(\mathrm{X})$ is integrably bounded and $\mathrm{F}_{\mathrm{n}}(\omega) \stackrel{K-M}{\rightarrow}$ $F(\omega) \mu-a \cdot e$.

$$
\text { then } \mathrm{S}_{\mathrm{F}_{\mathrm{n}}}^{1} \stackrel{\mathrm{K}-\mathrm{M}}{\rightarrow} \mathrm{s}_{\mathrm{F}}^{1}
$$

PROOF. Note that because for all $n \geq 1 F_{n}(\omega) \subseteq G(\omega) \mu-a . e$. with $G(\omega) \varepsilon P_{w k c}(X)$ $w-\overline{\lim } F_{n}(\omega) \neq \phi \mu-$ a.e. But $\omega-\overline{\operatorname{IIm}} F_{n}(\omega)=F(\omega) \neq \phi \mu-$.e.e. Also since $8-\underline{1 m} F_{n}(\omega)=$ $F(\omega) \mu-a . e$, we have that $F(\omega) \in P_{f c}(X) \mu-a . e$. and $\omega+F(\omega)$ is measurable (recall $\mu\left({ }^{\cdot}\right)$ is complete). So using theorems 4.1 and 4.2 , it is easy to see that $S_{F}^{1} \underline{K}_{n}-\underline{M}_{\rightarrow} s_{F}^{1}$.

Q.E.D.

We would like to have such a dominated convergence theorem for the Hausdorff mode of convergence. In this direction we have the following rusult. The spaces $(\Omega, \Sigma, \mu)$ and $X$ remain as before. 
THEOREM 4.5. If $F_{n}: \Omega \rightarrow P_{f c}(X)$ are measurable multifunctions s.t. $\left\{\left|F_{n}(\cdot)\right|\right\}_{n \geq 1}$ is uniformly integrable and $F_{n}(\omega) \rightarrow-\underline{h} \rightarrow F(\omega)$ in measure then $F: S a+P_{f c}(X)$ is integrably bounded and $S_{F_{n}}^{1} \stackrel{h}{\rightarrow} s_{F}^{1}$.

PROOF. First note that since $\left(P_{f c}(X), h\right)$ is a complete, metric space, we have

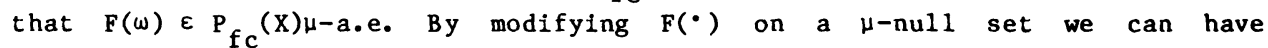
$F(\omega) \varepsilon P_{f c}(X)$ for all $\omega \in \Omega$ and since $\mu\left(^{\cdot}\right)$ is complete, the modified multifunction is still going to be measurable. Also from the properties of the Hausdorf $f$ metric we have that ||$F_{n}(\omega)|-| F(\omega)|| \leq h\left(F_{n}(\omega), F(\omega)\right) \mu-a_{. e .} \Rightarrow\left|F_{n}(\omega)\right|+|F(\omega)|$ in measure and since by hypothesis $\left\{F_{n}() \mid\right\}_{n \geq 1}$ is uniformly integrable, we deduce that $|F(\cdot)| \varepsilon L_{+}^{1}$ i.e. $F(\cdot)$ is integrably bounded as claimed by the theorem.

Next note that $\left\{s_{F_{n}}^{1}, s_{F}^{1}\right\}, 1$ are convex, closed and bounded subsets of $L_{x}^{1}(\Omega)$.

So recalling that $\left(\mathrm{L}_{\mathrm{X}}^{1}\right)^{*}=\mathrm{L}_{\mathrm{X}_{\mathrm{w}}^{*}}^{\infty}$ and using Hormander's formula we have that

$$
\begin{aligned}
& h\left(S_{F_{n}}^{1}, s_{F}^{1}\right)=\sup _{\|\left. u\right|_{\infty \leq 1}}\left|\sigma s_{F_{n}}^{1}(u)-\sigma_{s_{F}^{1}}(u)\right| \\
& \left.=\sup _{|| \mathrm{u}||_{\infty \leq 1}} \mid \int_{\Omega}\left(\sigma \quad \sigma_{\mathrm{n}}(\omega)(\omega)\right)-\sigma(\mathrm{u}(\omega))\right) \mathrm{d} \mu(\omega) \mid \\
& \leq \sup _{\left.|| u\right|_{\infty \leq 1}} \int_{\Omega}|\sigma| F_{n}(\omega)(\omega)-\sigma(u(\omega)) \mid d \mu(\omega)
\end{aligned}
$$

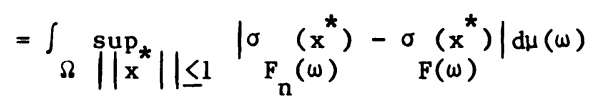

$$
\begin{aligned}
& =\int_{\Omega} h\left(F_{n}(\omega), F(\omega)\right) d \mu(\omega) .
\end{aligned}
$$

Since by hypothesis $\left\{\left|F_{n}\left(^{\cdot}\right)\right|\right\}_{n \geq 1}$ is uniformly integrable and $F_{n}(\omega)-\underline{h} \rightarrow F(\omega)$ in measure then $\int_{\Omega} h\left(F_{n}(\omega), F(\omega)\right) d \mu(\omega) \rightarrow 0 \Rightarrow h\left(S_{F_{n}}^{1}, s_{F}^{1}\right) \rightarrow 0$

Q.E.D.

We will conclude our work with an important observation about the Kuratowski-Mosco convergence of closed, convex sets. It is a very useful necessary condition for $K$ - $M$ convergence of such sets.

Assume that $X$ is a reflexive Banach space.

THEOREM 4.6. If $\left\{A_{n}\right\}_{n \geq 1} \subseteq P_{f c}(X), \sup _{n \geq 1}\left|A_{n}\right|<\infty$ and $A_{n} \stackrel{K-M}{\rightarrow} A$

$$
\text { then } A \neq \phi \text { and for a11 } x^{*} \varepsilon x^{*}, \sigma_{A}\left(x^{*}\right)+\sigma_{A}\left(x^{*}\right) \text {. }
$$

PROOF. Let $M=\sup _{n}\left|A_{n}\right|$ and let $B_{M}(0)$ be the $M$-ball centered at the origin. Then $B_{M}(0)$ is weakly ${ }^{n}$-compact and by the Eberlein-Smulian theorem sequentially w-compact. Let $x_{n} \in A_{n}, n \geq 1$. Then $\left\{x_{n}\right\}_{n \geq 1} \subseteq B_{m}(0)$ and so we can find a subsequence $\quad x_{k}-\underline{w} \rightarrow x$. Then $x \in w-\overline{1 i m} A_{n}=A \Rightarrow \not=\phi$. Next $f i x x^{*} \varepsilon x^{*}$ and let 
$x_{n} \varepsilon A_{n}$ s.t. $\left(x^{*}, x_{n}\right)=\sigma_{A_{n}}\left(x^{*}\right)$. By passing to an appropriate subsequence $\left\{x_{k}\right\} k \geq 1$

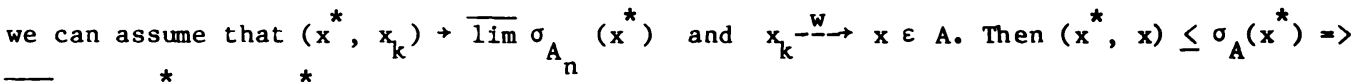
Iim $\sigma_{A_{n}}\left(x^{*}\right) \leq \sigma_{A}\left(x^{*}\right)$. On the other hand from Mosco [6] we know that $\sigma_{A_{n}}(\cdot)^{-} \stackrel{\tau}{\rightarrow} \sigma \sigma_{A}(\cdot)$ i.e. epi $\sigma_{A_{n}} \stackrel{K-M}{\rightarrow}$ epi $\sigma_{A}(\cdot)$ and this implies that $\underline{\lim } \sigma_{A_{n}}\left(x^{*}\right) \geq \sigma_{A}\left(x^{*}\right)[6]$ and $[7]$. So finally we have that $\sigma_{A_{n}}(\cdot) \rightarrow \sigma_{A}(\cdot)$.

Q.E.D.

REMARK. The converse of the above result is not true. Namely pointwise convergence of the support functions does not imply the Kurtowski-Mosco convergence of the corresponding closed, convex sets. Here is a counter example. Let $\left\{x_{n}\right\}_{n>1} \subseteq x$ and assume that $x_{n} \stackrel{w}{\rightarrow} x$ but it does not converge strongly. So $\left\{x_{n}\right\}$ do not converge to $\{x\}$ in the $K-M$ sense. On the other hand for every $x^{*} \varepsilon x^{*}\left(x^{*}, x_{n}\right)=$ $\sigma\left(x^{*}\right)+\left(x^{*}, x\right)=\sigma\left(x^{*}\right)$. So in corollary $2 E$ of $[10]$, it must be added that $x$ is $\left\{x_{n}\right\}$ $\{\mathbf{x}\}$

finite dimensional or otherwise the result is not true as the previous counterexample illustrated.

ACKNOWLEDGEMENT. This work was done while the author was visiting the Mathematics Department of the University of Pavia, Italy. Support was provided by C.N.R. and by N.S.F. Grant DMS-8403135. DMS-8602313.

\section{REFERENCES}

1. SChMeIdLeR, D. Fatou's Lemma in Several Dimensions, Proc. Amer. Math. Soc. 24 (1970), 300-306.

2. HILDENBRAND, W. and MERTENS, J.F. On Fatou's Lemma in Several Dimensions, Z. Wahrsch. verw. Gebiete 17 (1971), 151-155.

3. ARTSTEIN, Z. A Note on Fatou's Lemma in Several Dimensions, J. Math. Economics 6 $(1979), 277-282$.

4. KATO, T. Accretive Operators and Nonlinear Evolution Equations in Banach Spaces in the Proceedings of the Symposium on Nonlinear Functional Analysis, ed. F. Browder, AMS, Providence, RI, 1968, 138-161.

5. Mosco, U. Convergence of Convex Sets and of Solutions of Variational Inequalities, Advances in Math. $\underline{3}$ (1969), 510-585.

6. MOSC0, U. On the Continuity of the Young-Fenche1 Transform, J. Math. Ana1. App1. $35(1971), 518-535$.

7. SALINETTI, G. and WETS, R. On the Relations Between Two Types of Convergence for For Convex Functions, J. Math. Ana1. Appl. 60 (1977), 211-226.

8. SALINETTI, G. and WETS, R. On the Convergence of Sequences of Convex Sets in Finite Dimensions, SIAM Review 21 (1979), 18-33.

9 SALINETTI, G. and WETS, R. On the Convergence of Closed-valued Measurable Multifunctions, Trans. Ame r. Math. Soc. 266 (1981), 275-289.

10. PAPAGEORGIOU, N.S. Representations of Set Valued Operators, Trans. Amer. Math. Soc. $292(1986), 557-572$.

11. KURATOWSK I, K. Topology I, Academic Press, New York, (1966).

12. ASH, R. Real Analysis and Probability, Academic Press, New York (1972).

13. TSUKADA, M. Convergence of Best Approximations in a Smooth Banach Space, J. Approx. Th. 40 (1984), 301-309.

14. IONESCU-TULCEA, A. and C. Topics in the Theory of Lifting, Springer, Berlin (1969). 


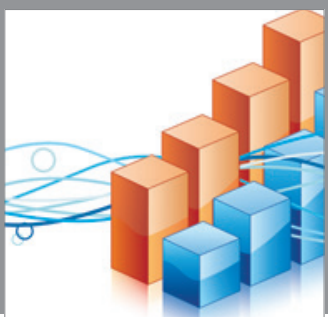

Advances in

Operations Research

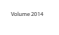

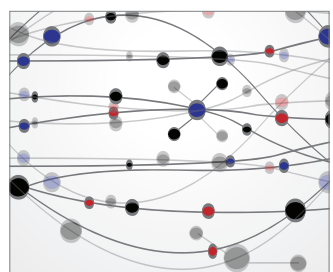

\section{The Scientific} World Journal
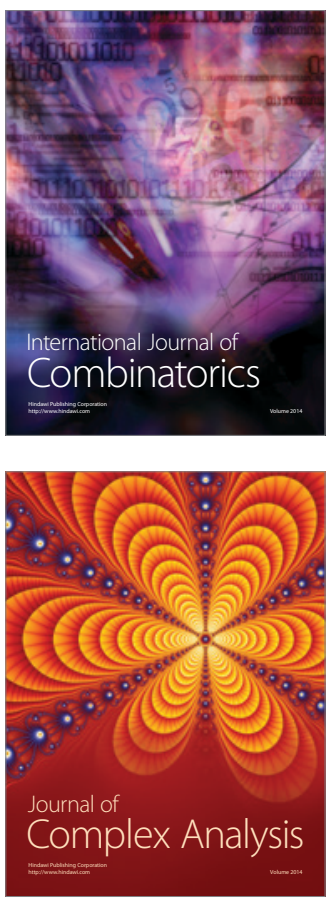

International Journal of

Mathematics and

Mathematical

Sciences
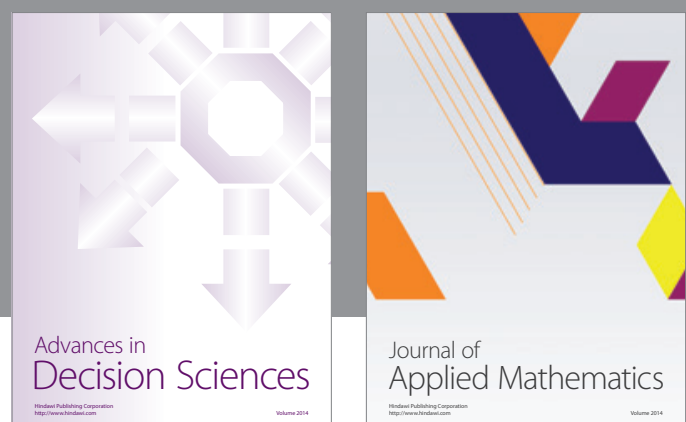

Journal of

Applied Mathematics
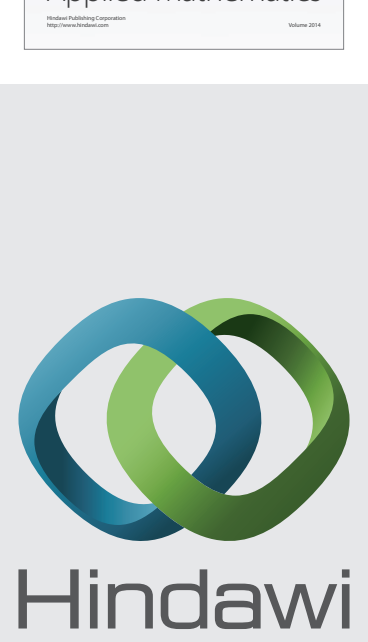

Submit your manuscripts at http://www.hindawi.com
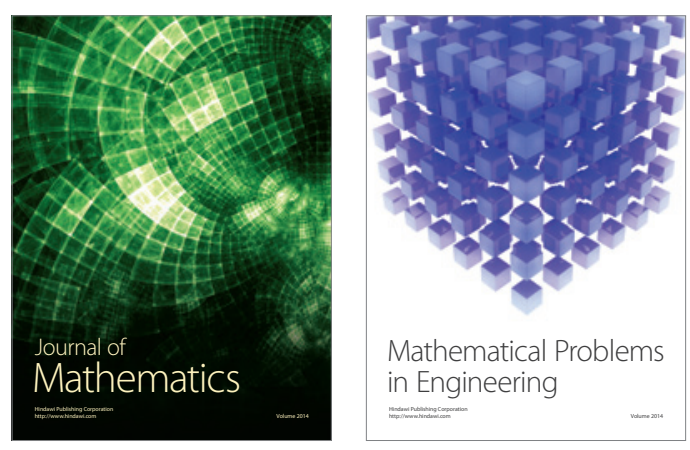

Mathematical Problems in Engineering
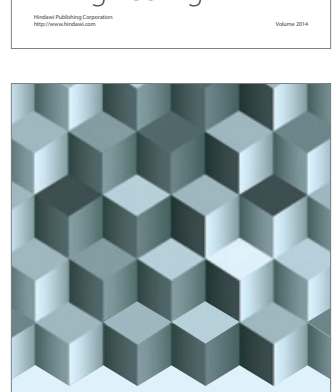

Journal of

Function Spaces
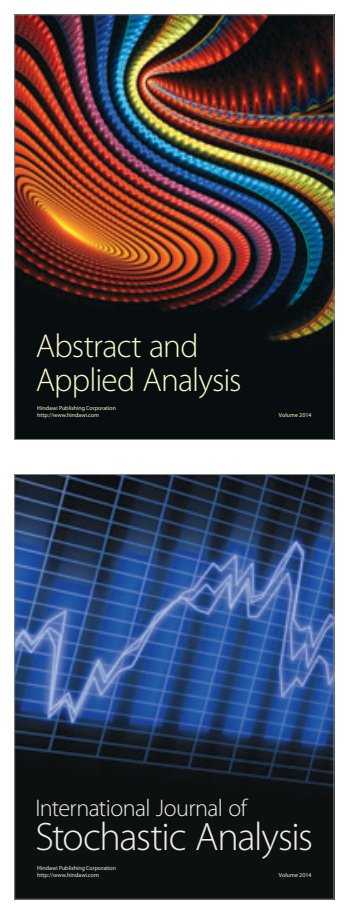

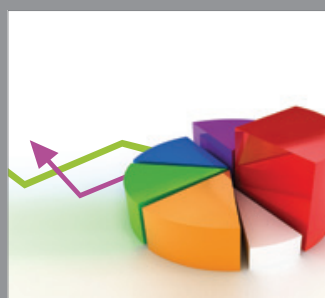

ournal of

Probability and Statistics

Promensencen
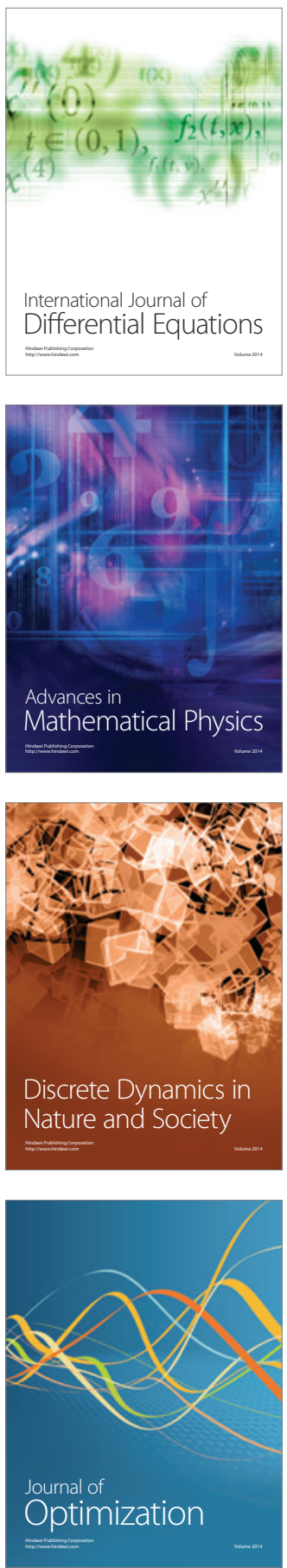